\title{
AVALIAÇÃO DA CONFIABILIDADE COMPOSTA SOB O ENFOQUE DO BEM ESTAR UTILIZANDO SIMULAÇÃO MONTE CARLO NÃO-SEQÜENCIAL
}

\author{
Tiago S. Amaral ${ }^{*}$
}

\author{
Carmen L.T. Borges ${ }^{\dagger}$
}

\author{
Andrea M. Rei* \\ ${ }^{*}$ CEPEL - Centro de Pesquisas de Energia Elétrica \\ Caixa Postal 68007 \\ CEP 21944-970 - Rio de Janeiro - RJ \\ ${ }^{\dagger}$ COPPE/UFRJ - Programa de Engenharia Elétrica \\ Caixa Postal 68504 \\ CEP 21941-972 - Rio de Janeiro - RJ
}

\section{RESUMO}

A avaliação da confiabilidade de sistemas de potência compostos têm sido realizada utilizando critérios e métodos determinísticos e probabilísticos. A avaliação da confiabilidade sob o enfoque do bem estar é uma nova proposta que combina critérios determinísticos com metodologias probabilísticas e define os estados do sistema como saudáveis, marginais ou de risco. Este trabalho apresenta a avaliação da confiabilidade composta sob o enfoque do bem-estar utilizando simulação Monte Carlo Não-Seqüencial. É assumida a hipótese de coerência do sistema e os índices de freqüência são calculados utilizando o método da probabilidade condicional. A avaliação do desempenho do sistema é feita através da solução do fluxo de potência pelo método de Newton-Raphson e da solução do fluxo de potência ótimo pelo método dos Pontos Interiores. São apresentados resultados para o sistema IEEE-RTS com e sem curva de carga. Demonstra-se que tanto a método escolhido como a hipótese assumida são válidos e fornecem uma alternativa eficiente para a análise

Artigo submetido em 19/06/2008 (Id.: 00878)

Revisado em 10/11/2008, 08/01/2009

Aceito sob recomendação do Editor Associado Prof. Julio Cesar Stacchini Souza do bem-estar de sistemas de grande porte.

PALAVRAS-CHAVE: Confiabilidade Composta, Enfoque do Bem Estar, Simulação Monte Carlo Não-Sequencial, Método da Probabilidade Condicional, Coerência.

\section{ABSTRACT}

The reliability evaluation of composite power systems has been historically assessed using deterministic and probabilistic criteria and methods. The well-being approach was recently proposed in order to combine deterministic criteria with probabilistic methods and evaluates the system by healthy, marginal and risky states. This paper presents the composite reliability evaluation by the well-being approach using the non-sequential Monte Carlo simulation. It is assumed that the system is coherent and the frequency and duration indices are calculated using the conditional probability method. The system adequacy is evaluated by the NewtonRaphson power flow method and the interior point optimal power flow method. Results are presented for the IEEE-RTS system with and without load curve. It is demonstrated that the evaluation method as well as the assumed hypothesis are valid and provides an efficient alternative for the well-being 
analysis of large scale power systems.

KEYWORDS: Composite Reliability, Well Being Analysis, Monte Carlo Simulation, Conditional Probability Method, Coherence.

\section{INTRODUÇÃO}

A avaliação da confiabilidade de sistemas elétricos de potência é comumente realizada em dois domínios, dependendo da forma como é feita a avaliação do desempenho do sistema: adequação e segurança. O domínio da adequação está relacionado com a existência de recursos suficientes dentro do sistema para satisfazer a demanda de carga e os seus requisitos operacionais. Esta análise está associada com condições estáticas que não incluem a dinâmica do sistema e a resposta a distúrbios transitórios. Já a análise no domínio da segurança está relacionada à habilidade do sistema responder a perturbações, tais como a perda de recursos de geração ou de transmissão e pode ser de dois tipos: transitória (dinâmica) ou de regime permanente (estática) (Billinton e Allan, 1996).

Por outro lado, a avaliação da confiabilidade pode ser realizada utilizando métodos determinísticos ou probabilísticos. Métodos determinísticos baseiam-se na avaliação de situações críticas a partir do histórico operativo e da experiência dos planejadores e operadores. Esses métodos têm características bastante atrativas, como a implementação direta e o fácil entendimento dos resultados obtidos. A maior fraqueza desses métodos é que não capturam a natureza estocástica da operação do sistema e podem levar a resultados muito conservativos ou pouco significativos (Allan e Billinton, 2000). Já os métodos probabilísticos, que são baseados na teoria de probabilidades e na estatística, levam em conta o comportamento aleatório do sistema, tais como o comportamento da carga e a disponibilidade de equipamentos de geração e transmissão. Esses métodos calculam índices de confiabilidade que são estimativas do risco associado ao sistema.

Dessa forma, há um considerável interesse em incluir aspectos de segurança estática em avaliações de adequação e em combinar considerações determinísticas com índices probabilísticos. Essa necessidade de aproximar as áreas de estudo da confiabilidade, melhorando o entendimento e a análise dos índices de confiabilidade, e possibilitando uma detecção prévia de estados de alerta do sistema, deu origem a proposta de avaliação da confiabilidade sob o enfoque do "bem-estar" do sistema.

Métodos probabilísticos foram inicialmente aplicados à avaliação da confiabilidade composta na década de 1960 (Billinton, 1969), sendo posteriormente desenvolvidos e consolidados dois métodos de avaliação da confiabilidade de sistemas compostos: a simulação Monte Carlo (SMC) e os métodos de enumeração. Desde então, a avaliação probabilística da confiabilidade composta ganhou cada vez mais interesse e diferentes formas de simulação foram propostas, dentre as quais destacam-se a SMC seqüencial e não-seqüencial (Pereira e Balu, 1992), (Billinton e Li, 1994), (Billinton e Li, 1993), a pseudo-seqüencial (Mello et alii, 1994) e a pseudocronológica (Leite da Silva et alii, 2000). Na aplicação da simulação não-seqüencial, têm destaque dois métodos de cálculo dos índices de frequiência e duração (F\&D): o método da probabilidade condicional (Melo et alii, 1993) e o método de transição "um passo a frente" (Manso e Leite da Silva, 2004).

Na década de 1990, foi desenvolvido um novo enfoque na avaliação da confiabilidade visando combinar critérios determinísticos com metodologias probabilísticas e incluir considerações de segurança estática em análises de adequação. Esse enfoque vem sendo chamada de "health analysis" (Billinton e Lian, 1994), (Billinton e Billinton, 1997), (Billinton et alii, 1997) ou "well-being analysis" (Goel e Feng, 1999), (Billinton e Karki, 1999), (Leite da Silva et alii, 2004), em inglês, e de "confiabilidade preventiva" (Manso et alii, 2004), em português.

A avaliação da confiabilidade sob o enfoque do bem-estar, como é chamada neste artigo, avalia a probabilidade não apenas de o sistema chegar a um estado de risco, mas também de estar muito perto dele. Isso é conseguido incluindo considerações determinísticas em avaliações probabilísticas. Nesse tipo de avaliação, diferentemente dos métodos tradicionais, os estados de sucesso são cuidadosamente avaliados para diferenciar entre estados que atendem (estados saudáveis) ou não (estados marginais) ao critério determinístico. Assim, esta abordagem fornece mais informações do que a tradicional, já que calcula não apenas o risco do sistema, mas também o grau de bem-estar do sistema baseado no critério determinístico adotado.

A confiabilidade sob o enfoque do bem-estar foi aplicada para sistemas de geração (Billinton e Karki, 1999), (Billinton e Billinton, 2005), para estudos de reserva operativa (Billinton e Fortuhi-Firubazad, 1994) e para confiabilidade composta, empregando técnicas de enumeração de estados (Billinton e Khan, 1992), (Billinton e Lian, 1994), (Goel e Feng, 1999) ou simulação Monte Carlo (Khan e Billinton, 1992). A aplicação em confiabilidade composta foi estendida para sistemas de grande porte em (Leite da Silva et alii, 2004), utilizando SMC não-seqüencial com o cálculo dos índices F\&D pelo método "um passo a frente". Mais recentemente, foi proposta uma abordagem utilizando SMC seqüencial para a avaliação do bem-estar de sistemas compostos (Wangdee e Billinton, 2006).

A principal questão envolvida com esse enfoque é o número excessivo de análises adicionais que são requeridas para di- 
ferenciar entre os estados saudáveis e marginais, o que exige grande esforço computacional, especialmente na confiabilidade composta. Dessa forma, um dos principais objetivos nos estudos recentes desse tema tem sido desenvolver métodos computacionalmente eficientes a fim de tornar viável sua aplicação a sistemas de grande porte. Em (Resende et alii, 2008) é proposta a aplicação de inteligência computacional baseada em redes neurais artificiais para acelerar a avaliação dos estados operativos de sistemas compostos. Outra forma de reduzir o esforço computacional é aplicando técnicas de processamento paralelo (Borges et alii, 2001), tendo em vista o elevado grau de desacoplamentos entre as análises de adequação necessárias.

Este artigo propõe um método de avaliação da confiabilidade composta sob o enfoque do bem-estar utilizando simulação Monte Carlo Não-Seqüencial, onde os índices F\&D são calculados utilizando o método da probabilidade condicional (Melo et alii, 1993). Para tanto, é assumida a hipótese de coerência no sistema. O objetivo desta proposta é tirar vantagem do menor esforço computacional requerido pela utilização do método da probabilidade condicional aliado à simulação Monte Carlo Não-Seqüencial, permitindo sua utilização em sistemas de grande porte. Esse esforço computacional deve ser menor que aquele gasto em (Leite da Silva et alii, 2004) devido à aplicação do método da probabilidade condicional, como será visto adiante. Além disso, deve ser ainda menor que aquele gasto em (Wangdee e Billinton, 2006), por causa da aplicação da simulação não-seqüencial em lugar da seqüencial.

\section{CONFIABILIDADE SOB O ENFOQUE DO BEM ESTAR}

O conceito da avaliação do bem-estar do sistema é uma estrutura probabilística que incorpora alguma consideração de segurança estática em avaliações de adequação. Para isso, utiliza um critério determinístico para avaliar se o sistema, quando em um estado de sucesso, está apto a suportar falhas sem violar qualquer restrição do sistema. Assim, o bem-estar do sistema pode ser categorizado em três estados, normalmente designados como: saudável, marginal e de risco (Billinton e Lian, 1994), conforme mostrado na Figura 1.

A cada etapa da avaliação probabilística, é aplicado o critério determinístico e o estado do sistema é classificado em uma das três categorias:

- estado de operação "saudável", em que o sistema opera dentro dos limites operativos sem corte de carga e atende ao critério determinístico;

- estado de operação "marginal", em que o sistema opera dentro dos limites operativos sem corte de carga, mas

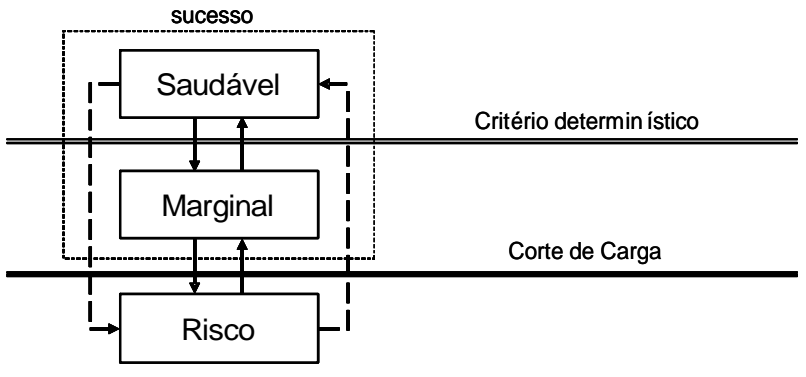

Figura 1: Diagrama de Estados no Enfoque do Bem-estar

não atende ao critério determinístico;

- estado "de risco", em que o sistema não opera dentro dos limites e há corte de carga.

Dessa forma, pode ser notado que a fronteira que separa os estados de sucesso (estados saudável e marginal) do estado de risco é o fato de haver ou não corte de carga. Por outro lado, a fronteira de separação entre os estados de sucesso é definida pelo atendimento ao critério determinístico. Como a fronteira entre estados de sucesso e de risco é o corte de carga, os índices de probabilidade, freqüência e duração do estado de risco são, respectivamente, os tradicionais índices LOLP, LOLF e LOLD. Portanto, a análise de bem-estar para sistemas compostos é uma extensão natural da análise de confiabilidade composta que permite a qualificação de um critério determinístico numa base probabilística.

Os estados marginais devem ser apropriadamente sinalizados a fim de dar aos operadores do sistema condições de corrigir a trajetória operativa do sistema e evitar o corte de carga (Leite da Silva et alii, 2004). Essa sinalização dos estados marginais fornecida pelo enfoque do bem-estar é uma preciosa informação que mede, em uma base probabilística, a percepção em relação ao grau de conforto da operação do sistema. A análise do bem-estar fornece uma interpretação quantitativa do grau de segurança (estado saudável) ou de insegurança (estado marginal) da operação do sistema. Por isso, um critério determinístico adequado é aquele que minimiza a possibilidade de o sistema operar em um estado de falha sem que o operador seja previamente alertado (Manso et alii, 2004). Isso significa que, se o critério determinístico é adequado, a transição entre estados saudáveis e estados de risco deve ser mínima. A escolha desse critério determinístico é de extrema importância para a análise sob enfoque do bem-estar pois é ele quem determina a separação entre o que é considerado um estado "saudável" do sistema e o que é considerado um estado "marginal".

Os índices do bem-estar podem incorporar qualquer critério determinístico especificado. Na avaliação da confiabilidade composta de sistemas de grande porte, em geral, são utiliza- 
das listas de contingências pré-estabelecidas. Essas listas de contingências podem ser vistas como uma variação do critério $\mathrm{N}-1$, nas quais apenas certos elementos são escolhidos para falhar. Os componentes da lista de contingência são baseados na experiência de operação e/ou no impacto no sistema. Naturalmente, o próprio critério $\mathrm{N}-1$ poderia ser utilizado, o que permitiria a detecção de contingências críticas a cada novo ponto de operação do sistema analisado. No entanto, isto não é normalmente realizado devido ao aumento do esforço computacional que seria necessário para tal avaliação.

Assim, com um critério determinístico, estados saudáveis são aqueles que sobrevivem, sem violações de limites operativos ou corte de carga, a todas as contingências da lista. Já os estados marginais são aqueles que não sobrevivem, sem violações ou corte de carga, a pelo menos uma das contingências.

O algoritmo geral da avaliação de confiabilidade sob enfoque do bem-estar está mostrado na Figura 2.

\subsection{Cálculo dos Índices de Probabilidade}

Os índices do bem-estar geralmente envolvem a probabilidade, a freqüência e a duração associados aos estados saudável, marginal e de risco. Estes índices são calculados através de funções teste. O cálculo dos índices de probabilidade é imediato. Se um dos três estados do sistema é identificado, esse cálculo pode ser feito através da função teste definida na Equação (1).

$$
\begin{aligned}
& F_{P(H)}\left(x_{i}\right)= \begin{cases}1 & \text { se } x_{i} \in \Omega_{H} \\
0 & \text { se } x_{i} \notin \Omega_{H}\end{cases} \\
& F_{P(M)}\left(x_{i}\right)= \begin{cases}1 & \text { se } x_{i} \in \Omega_{M} \\
0 & \text { se } x_{i} \notin \Omega_{M}\end{cases} \\
& F_{P(R)}\left(x_{i}\right)= \begin{cases}1 & \text { se } x_{i} \in \Omega_{R} \\
0 & \text { se } x_{i} \notin \Omega_{R}\end{cases}
\end{aligned}
$$

onde

$$
\begin{array}{ll}
\Omega_{H} & \begin{array}{l}
\text { conjunto de todos os estados saudáveis do sis- } \\
\text { tema } \\
\text { conjunto de todos os estados marginais do sis- } \\
\Omega_{M}
\end{array} \\
\Omega_{R} & \begin{array}{l}
\text { tema } \\
\text { conjunto de todos os estados de risco do sistema }
\end{array} \\
x_{i} & \text { estado } i \text { do sistema. } \\
P(H) & \text { probabilidade de estado saudável do sistema } \\
P(M) & \text { probabilidade de estado marginal do sistema } \\
P(R) & \text { probabilidade de estado de risco do sistema }
\end{array}
$$

Caso seja utilizada uma lista de contingências de tamanho $n_{l}$ como critério determinístico, podem ser necessárias até

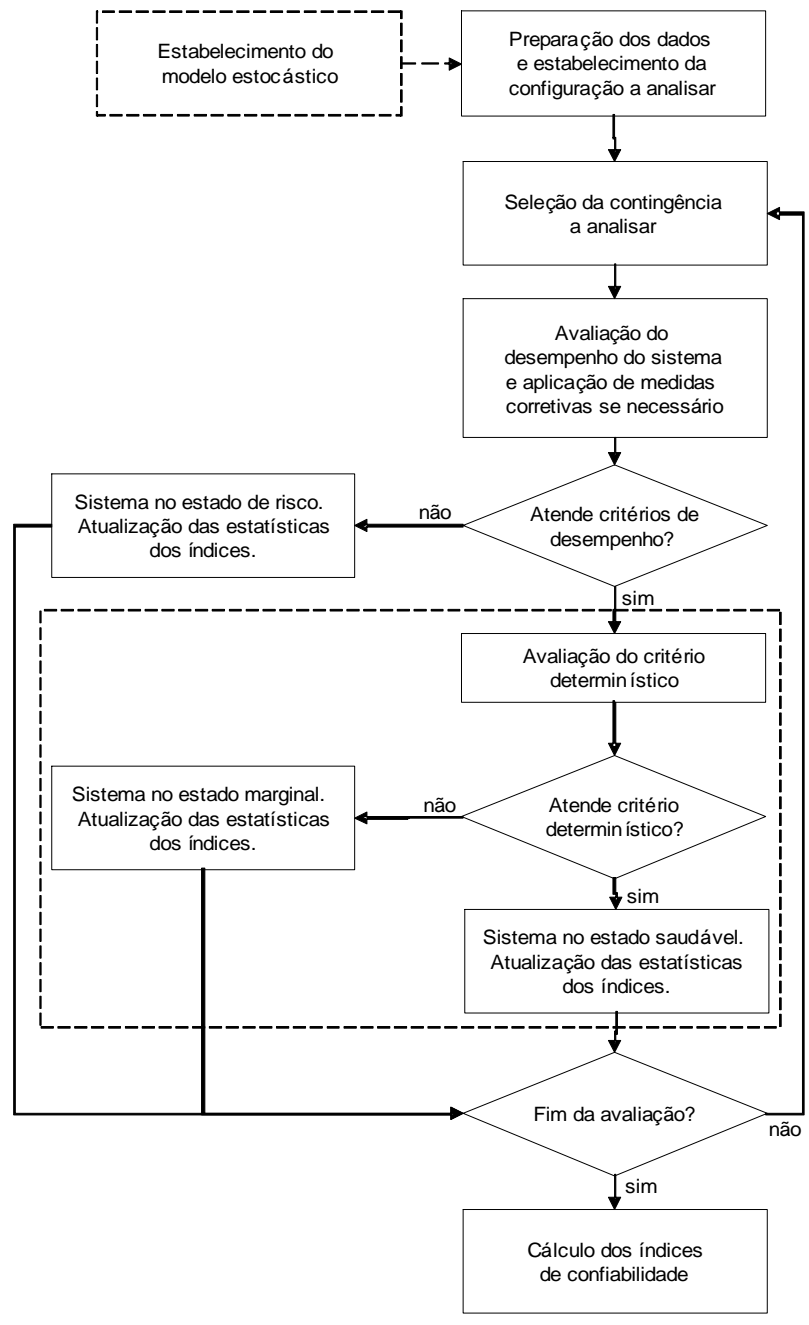

Figura 2: Algoritmo da Avaliação da Confiabilidade sob Enfoque do Bem-estar

$n_{l}$ análises de desempenho adicionais para distinguir entre os estados saudável e marginal. Numa avaliação de confiabilidade tradicional, para cada estado amostrado, apenas uma avaliação de desempenho é necessária para classificálo. Numa avaliação sob o enfoque de bem-estar, até $\left(n_{l}+1\right)$ avaliações de desempenho podem ser necessárias para classificar cada estado amostrado.

\subsection{Cálculo dos Índices de Freqüência}

Para o cálculo dos índices de freqüência, é necessário identificar as fronteiras que separam os diferentes estados do sistema, a fim de calcular a frequência com que são cruzadas. No enfoque do bem-estar, o sistema pode ser representado por um modelo de Markov a três estados, conforme mostrado na Figura 3. 


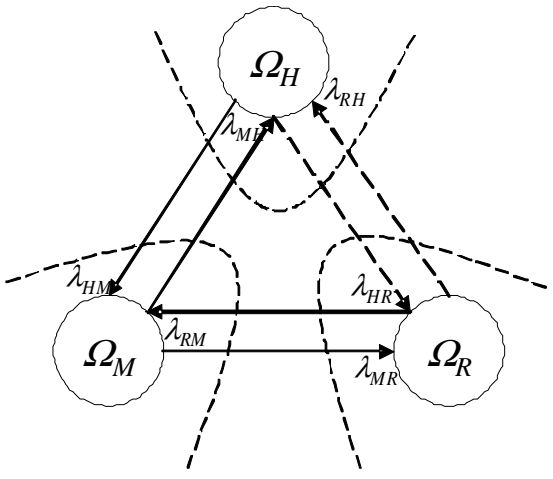

Figura 3: Sistema Modelado a Três Estados

\begin{tabular}{|c|c|}
\hline$I_{R}$ & $\begin{array}{l}\text { taxa de transição do subconjunto de estados sau- } \\
\text { dáveis para o de estados de risco }\end{array}$ \\
\hline$\lambda_{H M}$ & $\begin{array}{l}\text { taxa de transição do subconjunto de estados sau- } \\
\text { dáveis para o de estados marginais }\end{array}$ \\
\hline$\lambda_{M H}$ & $\begin{array}{l}\text { taxa de transição do subconjunto de estados } \\
\text { marginais para o de estados saudáveis }\end{array}$ \\
\hline$\lambda_{M R}$ & $\begin{array}{l}\text { taxa de transição do subconjunto de estados } \\
\text { marginais para o de estados de risco }\end{array}$ \\
\hline$\lambda_{R M}$ & $\begin{array}{l}\text { taxa de transição do subconjunto de estados de } \\
\text { risco para o de estados marginais }\end{array}$ \\
\hline$R H$ & $\begin{array}{l}\text { taxa de transição do subconjunto de estados de } \\
\text { risco para o de estados saudáveis }\end{array}$ \\
\hline
\end{tabular}

Supondo que o sistema seja ergódico, ou seja, sem estados absorventes, o sistema de equações (2) é válido:

$$
\left\{\begin{aligned}
F(H) & =P(H) \cdot \lambda_{H M}+P(H) \cdot \lambda_{H R} \\
& =P(M) \cdot \lambda_{M H}+P(R) \cdot \lambda_{R H} \\
F(M) & =P(M) \cdot \lambda_{M H}+P(M) \cdot \lambda_{M R} \\
& =P(H) \cdot \lambda_{H M}+P(R) \cdot \lambda_{R M} \\
F(R) & =P(R) \cdot \lambda_{R H}+P(R) \cdot \lambda_{R M} \\
& =P(H) \cdot \lambda_{H R}+P(M) \cdot \lambda_{M R}
\end{aligned}\right.
$$

onde

$F(H) \quad$ frequiência de ocorrência de estados saudáveis

$F(M) \quad$ frequiência de ocorrência de estados marginais

$F(R) \quad$ freqüência de ocorrência de estados de risco

Assim, os índices de frequiência podem ser calculados utilizando a função teste definida na Equação (3).

A avaliação dos parâmetros $\Delta \lambda_{M H}, \Delta \lambda_{R H}, \Delta \lambda_{M R}$ e $\Delta \lambda_{R M}$ é extremamente custosa para sistemas de grande porte. Para um sistema com $m$ componentes, seria necessário, a princípio, um número superior a $m$ avaliações de desempenho extras para calcular esses parâmetros para cada estado de risco ou marginal, além das avaliações necessárias para classificar o estado amostrado em saudável, marginal ou de risco (Manso et alii, 2004). Assim, a aplicação da função teste (3) não é indicada para sistemas reais de grande porte. Essa dificuldade estimulou o desenvolvimento de uma nova função teste baseada no método da probabilidade condicional, que será apresentada a seguir.

$$
\begin{aligned}
& F_{F(H)}\left(x_{i}\right)=\left\{\begin{array}{cl}
0, & \text { se } \mathrm{x}_{\mathrm{i}} \in \Omega_{\mathrm{H}} \\
\Delta \lambda_{M H}, & \text { se } \mathrm{x}_{\mathrm{i}} \in \Omega_{\mathrm{M}} \\
\Delta \lambda_{R H}, & \text { se } \mathrm{x}_{\mathrm{i}} \in \Omega_{\mathrm{R}}
\end{array}\right. \\
& F_{F(M)}\left(x_{i}\right)=\left\{\begin{array}{cl}
\Delta \lambda_{M H}+\Delta \lambda_{M R}, & \text { se } \mathrm{x}_{\mathrm{i}} \in \Omega_{\mathrm{M}} \\
0, & \text { se } \mathrm{x}_{\mathrm{i}} \notin \Omega_{\mathrm{M}}
\end{array}\right. \\
& F_{F(R)}\left(x_{i}\right)=\left\{\begin{array}{cc}
\Delta \lambda_{R H}+\Delta \lambda_{R M}, & \text { se } \mathrm{x}_{\mathrm{i}} \in \Omega_{\mathrm{R}} \\
0, & \text { se } \mathrm{x}_{\mathrm{i}} \notin \Omega_{\mathrm{R}}
\end{array}\right.
\end{aligned}
$$

onde

$\Delta \lambda_{M H} \quad$ somatório de todas as taxas de transição do estado $x_{i}$ marginal para todos os estados do subconjunto de estados saudáveis que possam ser obtidos a partir de $x_{i}$ em uma transição.

$\Delta \lambda_{R H} \quad$ somatório de todas as taxas de transição do estado $x_{i}$ de risco para todos os estados do subconjunto de estados saudáveis que possam ser obtidos a partir de $x_{i}$ em uma transição.

$\Delta \lambda_{M R} \quad$ somatório de todas as taxas de transição do estado $x_{i}$ marginal para todos os estados do subconjunto de estados de risco que possam ser obtidos a partir de $x_{i}$ em uma transição.

$\Delta \lambda_{R M} \quad$ somatório de todas as taxas de transição do estado $x_{i}$ de risco para todos os estados do subconjunto de estados marginal que possam ser obtidos a partir de $x_{i}$ em uma transição.

\subsection{Método Proposto para Cálculo dos Ín- dices de Freqüência}

O método da probabilidade condicional (Melo et alii, 1993) é um eficiente método para o cálculo dos índices de freqüência na simulação Monte Carlo não-seqüencial, porque não exige nenhuma avaliação de desempenho adicional além daquelas já necessárias para estimar os índices de probabilidade.

A única restrição deste método é a hipótese de coerência do sistema (Fardis e Cornell, 1981): se um componente falho é reparado, o desempenho do sistema nunca piora; por outro lado, se um componente que está operando falha, o desempenho do sistema nunca melhora. Já foi comentado na literatura (Melo et alii, 1993) que esta hipótese é sempre válida 
para geradores, tanto em análises de confiabilidade da geração como em análises de confiabilidade composta, quando um modelo de fluxo de potência linearizado é utilizado. Contudo, essa hipótese pode não ser válida em circuitos de transmissão, como foi discutido em (Melo et alii, 1992), que mostrou que, para os três sistemas analisados com um modelo de fluxo de potência linearizado, a não coerência do sistema tem um efeito desprezível nos índices de confiaiblidade do sistema.

A seguir são apresentadas as funções teste para cada um dos índices de frequiência de uma avaliação do bem-estar baseado no método da probabilidade condicional.

\subsubsection{Função Teste para $F(R)$}

A Figura 3 mostra que a fronteira entre estados de risco e estados de sucesso é a mesma fronteira que a avaliação de confiabilidade tradicional calcula. Dessa forma, para a avaliação do índice $F(R)$, a função teste utilizada pode ser a mesma utilizada no método da probabilidade condicional para o cálculo da LOLF, conforme mostrado na Equação (4.a).

$$
F_{F(R)}\left(x_{i}\right)=\left\{\begin{array}{cl}
0, & \text { se } x_{i} \notin \Omega_{R} \\
\sum_{k=1}^{m}\left[\lambda_{k}^{+}-\lambda_{k}^{-}\right], & \text {se } x_{i} \in \Omega_{R}
\end{array}\right.
$$

onde $m$ é o número de componentes do sistema, $\lambda_{k}^{+}$é a taxa de transição do componente $k$ para um estado "superior". No caso dos equipamentos, os estados superiores são estados de operação mais próximos da operação nominal. No caso da carga, são estados de carga mais baixa. Por outro lado, $\lambda_{k}^{-}$é a taxa de transição do componente $k$ para um estado "inferior". No caso dos equipamentos, os estados inferiores são estados de operação mais distantes da operação nominal. No caso da carga, são estados de carga mais alta.

Para componentes modelados a dois estados, essa equação pode ser reescrita como mostrado na Equação (4.b).

$$
F_{F(R)}\left(x_{i}\right)=\left\{\begin{array}{cl}
0, & \text { se } \mathrm{x}_{\mathrm{i}} \notin \Omega_{R} \\
\sum_{k \in D_{i}} \mu_{k}-\sum_{k \in U_{i}} \lambda_{k}, & \text { se } \mathrm{x}_{\mathrm{i}} \in \Omega_{R}
\end{array}\right.
$$

onde

$U_{i}$ conjunto de todos os componentes operando no estado $i$

$D_{i}$ conjunto de todos os componentes falhados no estado $i$

$\mu_{k}$ taxa de reparo do componente $k$

$\lambda_{k}$ taxa de falha do componente $k$

\subsubsection{Função Teste para $\mathbf{F}(\mathbf{H})$}

O cálculo do índice $F(H)$ poderia seguir um procedimento semelhante ao da Equação (4), porém com sinais invertidos, para todos os estados saudáveis encontrados. No entanto, o cálculo dos índices no sentido Falha-Sucesso é muito menos custoso e de convergência muito mais rápida do que no sentido inverso (Melo et alii, 1993). Em outras palavras, em vez de armazenar as estatísticas do índice $F(H)$ toda vez que ocorre um estado saudável, é proposto que as estatísticas desse índice sejam armazenadas toda vez em que ocorre um estado não saudável (marginal ou de risco), conforme a Equação (5).

$$
\begin{gathered}
F_{F(H)}\left(x_{i}\right)=\left\{\begin{array}{rr}
0, & \text { se } x_{i} \in \Omega_{H} \\
\sum_{k=1}^{m}\left[\lambda_{k}^{+}-\lambda_{k}^{-}\right], & \text {se } x_{i} \notin \Omega_{H}
\end{array}\right. \\
F_{F(H)}\left(x_{i}\right)=\left\{\begin{array}{rr}
0, & \text { se } x_{i} \in \Omega_{H} \\
\sum_{k \in D_{i}} \mu_{k}-\sum_{k \in U_{i}} \lambda_{k}, & \text { se } x_{i} \notin \Omega_{H}
\end{array}\right.
\end{gathered}
$$

\subsubsection{Função Teste para $F(M)$}

Não existe raciocínio semelhante aos anteriores que possa levar ao cálculo de $F(M)$. Por outro lado, o sistema de Equações (2) pode ser resolvido para encontrar $F(M)$. Somando a primeira e a terceira equações, chega-se a Equação (6).

$$
\begin{array}{r}
F(R)+F(H)=\left[P(M) \cdot \lambda_{M H}+P(M) \cdot \lambda_{M R}\right] \\
+\left[P(R) \cdot \lambda_{R H}+P(H) \cdot \lambda_{H R}\right] \\
=F(M)+\delta
\end{array}
$$

Na Equação (6), o primeiro termo entre colchetes é exatamente $F(M)$ enquanto que o segundo termo é definido aqui como um valor $\delta$, conforme mostrado na Equação (7).

$$
\delta=P(R) \cdot \lambda_{R H}+P(H) \cdot \lambda_{H R}
$$

Este valor $\delta$ é calculado por dois termos, sendo o primeiro função de $\lambda_{R H}$, a taxa de transição entre estados de risco e estados saudáveis, e o segundo de $\lambda_{H R}$, a taxa de transição entre estados saudáveis e estados de risco. Deve ser notado que a grande vantagem da estrutura de bem-estar é a identificação dos estados marginais, que assinalam os estados de alerta (inseguros) do sistema. Assim, um critério determinístico bem elaborado é aquele que apresenta a frequiência de transição entre os estados saudáveis e de risco mínima, para assegurar que os estados marginais serão corretamente assinalados. Isso faz com que o valor de $\delta$ seja muito próximo 
de zero. Se não fosse assim, a aplicação desse enfoque não teria sentido. Assim, pode ser concluído que o termo $\delta$ pode ser desprezado. Isso equivale a dizer que toda vez que o sistema tem que transitar entre os estados de risco e saudável, ele passará necessariamente pelo estado marginal.

Por esta proposta, como o valor do termo $\delta$ é sempre positivo, será obtido um valor de $F(M)$ conservativo, muito próximo e ligeiramente superior ao real. Dessa forma, a função teste para o cálculo de $F(M)$ é simplesmente a soma das funções teste anteriores, conforme mostrado na Equação (8).

$$
F_{F(M)}\left(x_{i}\right)=\left\{\begin{aligned}
0, & \text { se } x_{i} \in \Omega_{H} \\
\sum_{k=1}^{m}\left(\lambda_{k}^{+}-\lambda_{k}^{-}\right), & \text {se } x_{i} \in \Omega_{M} \\
2 \cdot\left[\sum_{k=1}^{m}\left(\lambda_{k}^{+}-\lambda_{k}^{-}\right)\right], & \text {se } x_{i} \in \Omega_{R}
\end{aligned}\right.
$$

$$
F_{F(M)}\left(x_{i}\right)=\left\{\begin{aligned}
0, & \text { se } x_{i} \in \Omega_{H} \\
\sum_{k \in D_{i}} \mu_{k}-\sum_{k \in U_{i}} \lambda_{k}, & \text { se } x_{i} \in \Omega_{M}
\end{aligned}\right.
$$

Com esse método proposto, não é necessária nenhuma simulação extra a partir do estado atual e o esforço computacional para o cálculo dos índices de frequiência permanece equivalente ao esforço já utilizado para o cálculo dos índices de probabilidade.

\section{MODELO COMPUTACIONAL}

Para a implementação da avaliação da confiabilidade composta sob enfoque do bem-estar, foi desenvolvido um modelo computacional na linguagem de programação Lua (Ierusalimschy et alii, 2006), denominado PS/Lua. Lua é uma linguagem de programação baseada em script projetada para suportar programação procedural, com facilidades para descrição de dados. O modelo desenvolvido, PS/Lua, é composto por:

- uma biblioteca de vínculo dinâmico (dynamic link library) contendo parte do código do programa $\mathrm{NH} 2{ }^{\mathrm{R}}$, desenvolvido pelo CEPEL, versão 8.0 (CEPEL, 2006);

- uma biblioteca contendo funções escritas em $\mathrm{C}$ para tornar acessíveis ao modelo PS/Lua as principais funcionalidades do programa $\mathrm{NH} 2$, em especial, a função de solução de contingências;
- uma biblioteca escrita em Lua contendo a implementação de diversos algoritmos de avaliação da confiabilidade composta, tais como SMC sequencial, SMC nãosequencial, avaliação sob o enfoque do bem estar, etc.

A figura 4 mostra o esquema de interação entre o PS/Lua e os módulos do $\mathrm{NH}_{2}{ }^{\circledR}$. Dessa forma, o programa PS/Lua utiliza os métodos numéricos implementados no $\mathrm{NH} 2{ }^{\circledR}$ e os acessa através de uma linguagem de script, oferecendo um maior grau de liberdade para automatizar tarefas, criar modelos e realizar estudos profundos. Dessa forma, a precisão e eficiência dos métodos de solução de um programa comercial amplamente utilizado e validado, como o $\mathrm{NH} 2{ }^{\circledR}$, são preservados.

A avaliação do desempenho de cada estado do sistema é feita através da solução do fluxo de potência não-linear pelo método de Newton-Raphson, seguido da aplicação de medidas corretivas através da solução do fluxo de potência ótimo pelo método dos Pontos Interiores (Granville, 1994).

\section{RESULTADOS}

A fim de validar o método proposto e compará-lo com os demais métodos reportados na literatura, foram realizadas diversas análises com o sistema teste IEEE-MRTS. O sistema IEEE-MRTS é idêntico ao sistema IEEE-RTS (IEEE Task Force, 1979), exceto que a capacidade da geração e a carga de todas as barras são dobradas, estressando a rede de transmissão. Assim, a capacidade de geração instalada é de 6810 MW e 3552 MVAr e o pico de carga é de 5700 MW e 1160 MVAr. O diagrama unifilar do sistema está mostrado na Fi-

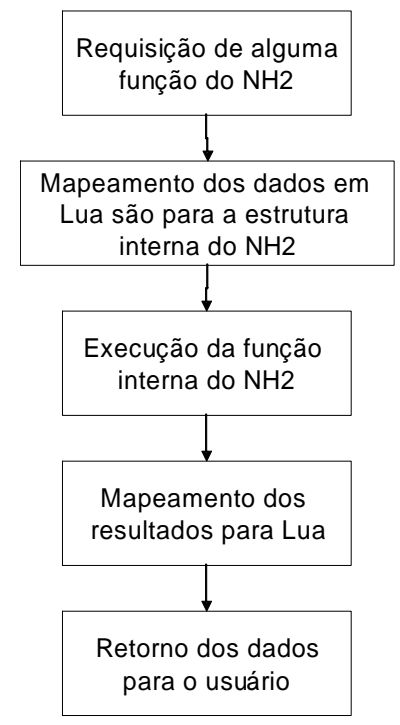

Figura 4: Fluxograma de interação PS/Lua-NH2 
gura 5 .

Para esse sistema, dois tipos de estudo foram considerados:

- Caso1: Sem curva de carga, isto é, carga em todas as barras igual ao pico anual de carga.

- Caso 2: Com curva de carga da semana de pico, isto é, carga em todas as barras com variação anual igual a 52 repetições da semana de pico (semana 51 de (IEEE Task Force, 1979)).

Para cada caso, foi realizada a avaliação da confiabilidade composta usando o enfoque probabilístico tradicional e o enfoque de bem estar. Para cada enfoque, foram utilizados três métodos distintos de simulação, a saber:

1. Método 1: SMC Seqüencial;

2. Método 2: SMC Não-Sequiencial com cálculo dos índices F\&D pelo método "um passo a frente";

3. Método 3: SMC Não-Sequiencial com cálculo dos índices F\&D pelo método da probabilidade condicional (método proposto).

Em todas as simulações, foi adotado como regra de parada o coeficiente de variação das estimativas dos índices $\beta \leq 5 \%$ para todos os índices. É importante salientar que em todos os métodos de simulação e enfoques, a análise da adequação dos estados amostrados foi realizada utilizando as mesmas rotinas de solução de fluxo de potência e fluxo de potência ótimo. As simulações foram realizadas em um computador baseado no processador Pentium IV 3.0GHz com $512 \mathrm{MB}$ de memória RAM.

\subsection{Caso 1: Sem curva de carga}

\subsubsection{Enfoque do Bem-Estar}

Para avaliação da confiabilidade do sistema pelo enfoque do bem-estar é necessária a utilização de um critério determinístico. Como já comentado, a adoção de um critério determinístico apropriado é de extrema importância para a análise sob enfoque do bem-estar.

No presente estudo, foi utilizado como critério uma lista de contingências críticas. Para a elaboração desta, podem ser utilizadas diferentes metodologias, como a avaliação de índices de severidade associados a violações nas condições operativas, por exemplo. Neste estudo, foi adotada a lista definida na Tabela 1 que está baseada na lista de contingências apresentada em (Leite da Silva et alii, 2004). Esta lista de

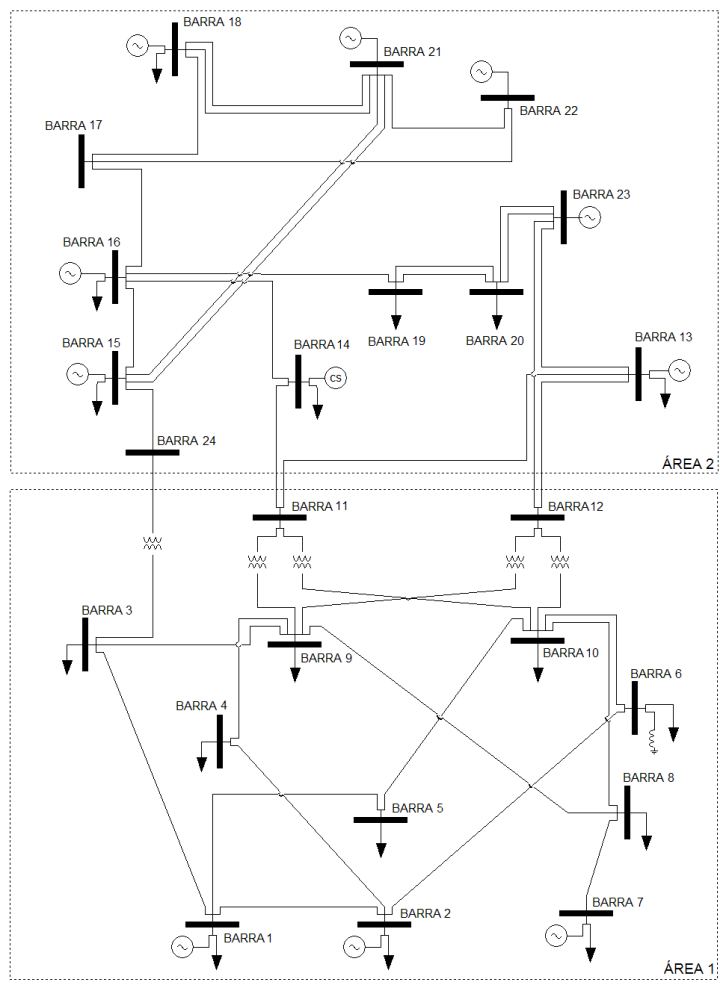

Figura 5: Diagrama Unifilar do Sistema IEEE-RTS

contingências foi utilizada a título de comparação entre os métodos avaliados e por se tratar do mesmo sistema teste. Foi excluída da lista de (Leite da Silva et alii, 2004) a avaliação do próximo patamar da curva de carga, devido ao modelo de carga adotado no método 3, como será descrito adiante. Deve ser notado que, embora a lista dessa referência tenha sido elaborada utilizando uma modelagem de fluxo de potência linear (DC), a mesma lista produziu resultados adequados também na modelagem apresentada no presente artigo. Os índices calculados estão mostrados na Tabela 2. O valor entre parenteses corresponde ao coeficiente de variação do índice calculado.

Pode ser observado o ganho de informação obtido com o enfoque do bem-estar. A partir de uma avaliação tradicional, poderia ser pensado que o sistema permanecesse cerca de $88 \%$ do tempo $(100-\mathrm{P}(\mathrm{R}))$ num estado confortável. No entanto, com o enfoque do bem-estar, observa-se que esse sistema permanece apenas cerca de $40 \%$ do tempo no estado saudável. Além disso, pode ser observado que o sistema entrará em estado inseguro aproximadamente 89 vezes durante o ano, o que é um número bastante alto.

O método da probabilidade condicional apresentou resultados equivalentes aos demais métodos. Em especial, o método proposto para o cálculo do índice $F(M)$ forneceu um resultado bastante próximo daquele fornecido pela simula- 
Tabela 1: Lista de Contingências

\begin{tabular}{|c|}
\hline $\begin{array}{c}\text { Lista de } \\
\text { Contingências }\end{array}$ \\
\hline Circuito 14-16 \\
\hline Circuito 13-11 \\
\hline Circuito 10-11 \\
\hline Circuito 10-12 \\
\hline Circuito 9-12 \\
\hline Circuito 23-12 \\
\hline Circuito 14-11 \\
\hline Circuito 9-11 \\
\hline Circuito 13-12 \\
\hline Usinas de 1 a 14 \\
\hline
\end{tabular}

ção seqüencial. A diferença entre os índices de frequência calculados pelo método proposto e aqueles calculados pelos demais métodos está mostrada na Tabela 3 , onde o valor entre parenteses representa a diferença percentual tomando como base os índices obtidos pelos outros métodos.

Tabela 2: Índices de Confiabilidade - Caso 1

\begin{tabular}{|l|l|l|l|}
\hline Índice & Método 1 & Método 2 & Método 3 \\
\hline $\begin{array}{l}\mathrm{P}(\mathrm{R}) \\
{[\%]}\end{array}$ & $\begin{array}{l}11.9484 \\
(4.97)\end{array}$ & $\begin{array}{l}12.4337 \\
(2.61)\end{array}$ & $\begin{array}{l}12.4878 \\
(3.12)\end{array}$ \\
\hline $\mathrm{P}(\mathrm{M})$ & 47.0381 & $\begin{array}{l}48.6737 \\
(1.01)\end{array}$ & $\begin{array}{l}48.0045 \\
(1.23)\end{array}$ \\
{$[\%]$} & $(1.33)$ & $\begin{array}{l}38.8926 \\
(1.23)\end{array}$ & $\begin{array}{l}39.5077 \\
(1.46)\end{array}$ \\
\hline $\begin{array}{l}\mathrm{P}(\mathrm{H}) \\
{[\%]}\end{array}$ & $\begin{array}{l}41.0135 \\
(2.07)\end{array}$ & $\begin{array}{l}27.5124 \\
(5.00)\end{array}$ & $\begin{array}{l}30.5508 \\
(5.00)\end{array}$ \\
\hline $\begin{array}{l}\mathrm{F}(\mathrm{R}) \\
{[\text { oc/ano] }}\end{array}$ & $\begin{array}{l}27.3030 \\
(2.95)\end{array}$ & $\begin{array}{l}89.1137 \\
(3.87)\end{array}$ \\
\hline $\begin{array}{l}\mathrm{F}(\mathrm{M}) \\
{[\mathrm{oc} / \mathrm{ano}]}\end{array}$ & $\begin{array}{l}88.7879 \\
(1.11)\end{array}$ & $\begin{array}{l}90.8019 \\
(2.21)\end{array}$ & $\begin{array}{l}58.5629 \\
(3.86)\end{array}$ \\
\hline $\begin{array}{l}\mathrm{F}(\mathrm{H}) \\
{[\text { oc/ano] }}\end{array}$ & $\begin{array}{l}62.1515 \\
(1.86)\end{array}$ & $\begin{array}{l}60.5178 \\
(2.80)\end{array}$ & 35.807 \\
\hline $\begin{array}{l}\mathrm{D}(\mathrm{R}) \\
{[\mathrm{h} / \mathrm{oc} .]}\end{array}$ & 38.336 & 39.589 & 47.189 \\
\hline $\begin{array}{l}\mathrm{D}(\mathrm{M}) \\
{[\mathrm{h} / \mathrm{oc} .]}\end{array}$ & 46.409 & 46.957 & 59.097 \\
\hline $\begin{array}{l}\mathrm{D}(\mathrm{H}) \\
{[\mathrm{h} / \mathrm{oc} .]}\end{array}$ & 57.807 & 56.297 & 7191 \\
\hline $\begin{array}{l}\text { Estados } \\
\text { Simula- } \\
\text { dos }\end{array}$ & $\begin{array}{l}16125 \\
(33 \text { anos })\end{array}$ & 10367 & \\
\hline \begin{tabular}{l} 
Tempo \\
\hline
\end{tabular} & $31 \mathrm{~min} 50 \mathrm{~s}$ & $20 \mathrm{~min} 23 \mathrm{~s}$ & $8 \mathrm{~min} 31 \mathrm{~s}$ \\
\hline
\end{tabular}

Tabela 3: Diferença para Demais Métodos - Caso 1

\begin{tabular}{|l|l|l|}
\hline Índice & Diferença para Método 1 & Diferença para Método 2 \\
\hline $\mathrm{F}(\mathrm{R})$ & $3.24780(11.89 \%)$ & $3.0384(11.04 \%)$ \\
\hline $\mathrm{F}(\mathrm{M})$ & $0.32580(0.37 \%)$ & $1.6882(1.86 \%)$ \\
\hline $\mathrm{F}(\mathrm{H})$ & $3.58860(5.77 \%)$ & $1.9549(3.23 \%)$ \\
\hline
\end{tabular}

Pode ser observado que as diferenças apresentadas são pequenas, com um desvio médio de aproximadamente $6 \% \mathrm{em}$ relação a simulação sequencial, sendo a maior diferença de $11.89 \%$ no índice $F(R)$. A título de comparação, para se obter a mesma confiança para a estimativa deste índice obtida pela simulação sequencial ( $\beta=2.95 \%$ ), é necessário um aumento no número de estados avaliados e, consequentemente, no tempo de simulação. Neste caso, os índices obtidos pela método proposto passam a ser $F(R)=27.64 \mathrm{oc} / \mathrm{ano}$, $F(M)=90.89$ oc/ano e $F(H)=63.25$ oc/ano, todos com diferenças em relação à simulaçao sequencial dentro da margem de incerteza especificada para o parâmetro $\beta(5 \%)$.

Do ponto de vista de desempenho computacional, o tempo de processamento gasto pela método proposto foi inferior ao tempo gasto pelos outros métodos. O tempo demandado foi $42 \%$ do tempo da simulação com o método 2 e $27 \%$ do tempo com o método 1. Isso equivale a dizer que o método proposto foi 2,4 vezes mais rápido que o método "um passo a frente" e 3,7 vezes mais rápido que a simulação sequencial.

\subsubsection{Aplicabilidade do Método Proposto}

O método proposto para cálculo dos índices F\&D no enfoque de bem estar está baseada em duas hipóteses:

- Coerência do sistema;

- Frequiência de transição entre os estados de risco e saudável bastante pequena.

Para atestar a aplicabilidade desse método, ambas as hipóteses foram avaliadas utilizando a simulação seqüencial. Para avaliar a primeira hipótese, foram contadas todas as transições incoerentes que ocorreram na simulação seqüencial, da seguinte forma:

- se a quantidade de componentes em contingência aumentava, eram contadas todas as transições para estados "superiores", isto é, mais próximos do estado saudável, de acordo com a ordem da Figura 1;

- se diminuía, eram contadas todas as transições para estados "inferiores", isto é, mais próximos do estado de risco, de acordo com a ordem da Figura 1. 
Esses resultados são mostrados na Tabela 4, onde os números entre parênteses representam a porcentagem do número de transições incoerentes em relação ao número total de transições. Para avaliar a segunda hipótese, foi também calculado o termo $\delta$, representativo das freqüências de transição entre estados saudável e de risco.

Tabela 4: Aplicabilidade do Método Proposto - Caso 1

\begin{tabular}{|c|c|c|}
\hline \multicolumn{2}{|c|}{ Número de estados simulados } & 16125 \\
\hline \multicolumn{2}{|c|}{ Número total de transições } & 5882 \\
\hline \multirow{6}{*}{ 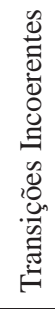 } & $R \Rightarrow M$ & $0(0.000 \%)$ \\
\hline & $R \Rightarrow H$ & $2(0.034 \%)$ \\
\hline & $M \Rightarrow H$ & $6(0.102 \%)$ \\
\hline & $H \Rightarrow M$ & $0(0.000 \%)$ \\
\hline & $H \Rightarrow R$ & $0(0.000 \%)$ \\
\hline & $M \Rightarrow R$ & $0(0.000 \%)$ \\
\hline \multicolumn{2}{|c|}{$\delta$ [oc/ano] } & 0.6667 \\
\hline
\end{tabular}

Pode ser notado que, neste caso, apenas $0.134 \%$ das transições foram incoerentes. Isso mostra que a hipótese de coerência é válida. Além disso, o valor do termo $\delta$ representa apenas $0.75 \%$ do valor do índice $F(M)$ calculado pela simulação seqüencial, o que ainda é bem menor que a incerteza relativa (5\%), atestando os bons resultados apresentados pelo método proposto.

Em resumo, a análise da aplicabilidade do método fornece duas conclusões:

- o critério determinístico foi apropriadamente escolhido, devido ao baixo valor do termo $\delta$;

- o fato de desprezar esse valor não teve grande impacto no cálculo dos índices de frequiência, considerada a incerteza na estimativa dos mesmos.

Assim, pode ser concluído que ambas as hipóteses são válidas para este caso e o método proposto é adequado. Deve ser observado que essas conclusões aplicam-se a este sistema específico. O sistema IEEE-RTS tem rede robusta e unidades de porte elevado comparadas à carga do sistema. É interessante repetir os testes de aplicabilidade a outros sistemas, com características distintas deste.

\subsection{Caso 2: Com curva de carga}

\subsubsection{Enfoque do Bem Estar}

Para avaliação da confiabilidade sob o enfoque do bem-estar considerando a curva de carga do sistema, foi utilizada uma
Tabela 5: Índices de Confiabilidade - Caso 2

\begin{tabular}{|l|l|l|l|}
\hline Índice & Método 1 & Método 2 & Método 3 \\
\hline $\begin{array}{l}\mathrm{P}(\mathrm{R}) \\
{[\%]}\end{array}$ & $\begin{array}{l}1.9204 \\
(4.99)\end{array}$ & $\begin{array}{l}1.9859 \\
(2.09)\end{array}$ & $\begin{array}{l}2.0010 \\
(2.97)\end{array}$ \\
\hline $\begin{array}{l}\mathrm{P}(\mathrm{M}) \\
{[\%]}\end{array}$ & $\begin{array}{l}11.9814 \\
(1.85)\end{array}$ & $\begin{array}{l}11.8226 \\
(0.81)\end{array}$ & $\begin{array}{l}11.7879 \\
(1.16)\end{array}$ \\
\hline $\begin{array}{l}\mathrm{P}(\mathrm{H}) \\
{[\%]}\end{array}$ & $\begin{array}{l}86.0982 \\
(0.32)\end{array}$ & $\begin{array}{l}86.1915 \\
(0.12)\end{array}$ & $\begin{array}{l}86.2111 \\
(0.17)\end{array}$ \\
\hline $\begin{array}{l}\mathrm{F}(\mathrm{R}) \\
{[\text { oc/ano }]}\end{array}$ & $\begin{array}{l}30.4083 \\
(3.82)\end{array}$ & $\begin{array}{l}33.4333 \\
(5.00)\end{array}$ & $\begin{array}{l}32.9651 \\
(4.99)\end{array}$ \\
\hline $\begin{array}{l}\mathrm{F}(\mathrm{M}) \\
{[\text { oc/ano }]}\end{array}$ & $\begin{array}{l}192.38 \\
(1.59)\end{array}$ & $\begin{array}{l}201.55 \\
(2.01)\end{array}$ & $\begin{array}{l}198.32 \\
(2.51)\end{array}$ \\
\hline $\begin{array}{l}\mathrm{F}(\mathrm{H}) \\
{[\text { oc/ano] }}\end{array}$ & $\begin{array}{l}163.45 \\
(1.34)\end{array}$ & $\begin{array}{l}171.82 \\
(2.18)\end{array}$ & $\begin{array}{l}165.36 \\
(2.48)\end{array}$ \\
\hline $\begin{array}{l}\mathrm{D}(\mathrm{R}) \\
{[\mathrm{h} / \mathrm{oc} .]}\end{array}$ & 5.5323 & 5.2033 & 5.3174 \\
\hline $\begin{array}{l}\mathrm{D}(\mathrm{M}) \\
{[\mathrm{h} / \mathrm{oc} .]}\end{array}$ & 5.4557 & 5.1385 & 5.2068 \\
\hline $\begin{array}{l}\mathrm{D}(\mathrm{H}) \\
{[\mathrm{h} / \mathrm{oc} .]}\end{array}$ & 46.1438 & 43.9435 & 45.6706 \\
\hline $\begin{array}{l}\text { Estados } \\
\text { Simula- } \\
\text { dos }\end{array}$ & $\begin{array}{l}1080945 \\
(120 \text { anos })\end{array}$ & 112894 & 55472 \\
\hline $\begin{array}{l}\text { Tempo } \\
15 \mathrm{~h} 39 \mathrm{~min}\end{array}$ & $\begin{array}{l}8 \mathrm{~h} 36 \mathrm{~min} \\
26 \mathrm{~s}\end{array}$ & $\begin{array}{l}3 \mathrm{~h} \\
50 \mathrm{~s}\end{array}$ \\
\hline
\end{tabular}

curva de carga formada por 52 repetições da semana de pico (semana 51) de (IEEE Task Force, 1979). A representação dessa curva de carga foi realizada diretamente nos métodos 1 e 2. Para o método 3, foi utilizado o modelo de carga agregado a múltiplos estados. Para tanto, foi realizado o agrupamento dos estados semelhantes da carga, totalizando 168 estados de carga distintos. Para realizar esse agrupamento, foi utilizada a metodologia detalhada em (Melo, 1990).

Além disso, foi utilizada a mesma lista de contingências definida na Tabela 1. Isso foi feito a fim de avaliar o efeito de manter a mesma lista e variar a representação da carga. Um outro enfoque, não adotado neste trabalho, poderia ser ter listas diferentes associadas a cada modelagem da carga. Os índices calculados estão mostrados na Tabela 5.

A partir desses resultados pode ser notado que, com a representação da carga, a permanência desse sistema no estado de risco diminui drasticamente, além de provocar forte impacto nos índices de estado marginal. Esse sistema agora permanece cerca de $86 \%$ do tempo no estado saudável e estará em estado inseguro por aproximadamente $12 \%$ do tempo 
Tabela 6: Diferença para Demais Métodos - Caso 2

\begin{tabular}{|l|l|l|}
\hline Índice & Diferença para Método 1 & Diferença para Método 2 \\
\hline $\mathrm{F}(\mathrm{R})$ & $2.5568(8.41 \%)$ & $0.4682(1.40 \%)$ \\
\hline $\mathrm{F}(\mathrm{M})$ & $5.9400(3.09 \%)$ & $3.2268(1.60 \%)$ \\
\hline $\mathrm{F}(\mathrm{H})$ & $1.9100(1.16 \%)$ & $6.4649(3.76 \%)$ \\
\hline
\end{tabular}

restante, o que pode ser visto como uma operação muito mais confortável que no caso anterior. Deve ser notado que o sistema entra no estado saudável cerca de 170 vezes, que é quase metade dos dias do ano. A duração média do estado saudável é de 46.14 horas, ou seja, o sistema permanece em estado saudável por esse período em média antes de transitar para o estado marginal, o que ocorre cerca de 200 vezes durante o ano. A diferença entre os índices de frequência calculados pelo método proposto em relação aos demais métodos está mostrada na Tabela 6.

O método proposto apresentou resultados bastante semelhantes aos da simulação sequiencial. As diferenças apresentadas são pequenas, com um desvio médio de aproximadamente $4.22 \%$, sendo a maior diferença de $8.41 \%$ no índice $F(R)$. A título de comparação, com a mesma confiança para a estimativa deste índice obtida pela simulação sequencial $(\beta=3.82 \%)$, os índices obtidos pela método proposto passam a ser $F(R)=31.86$ oc/ano, $F(M)=196$ oc/ano e $F(H)=164.13$ oc/ano, todos dentro da margem de incerteza especificada para $\beta$. A maior diferença em valor absoluto ficou por conta do índice $F(M)$. Essa diferença, no entanto, representa apenas cerca de $3.09 \%$ do índice calculado pela simulação seqüencial. O índice com a menor incerteza relativa foi $P(H)$, cuja diferença foi de $0.13 \%$ em relação ao índice calculado pela simulação seqüencial. Um observação importante é que, os índices calculados pelo método proposto para este caso estão mais próximos aos calculados pela simulação sequencial do que aqueles calculados pelo método 2 , que não adota a hipótese de coerência.

Dessa forma, pode ser percebido que com a inclusão da modelagem da variação na carga, o método proposto continuou válido e com resultados muito bons em termos de precisão.

O tempo computacional demandado pelo método proposto foi $46 \%$ do tempo do método 2 e $25 \%$ do tempo do método 1. Ou seja, o método proposto foi 2,2 vezes mais rápido que o método "um passo a frente" e quase 4 vezes mais rápido que a simulação seqüencial.

\subsubsection{Aplicabilidade do Método Proposto}

A avaliação da aplicabilidade do método proposto foi realizada da mesma forma que no caso anterior. Os resultados estão mostrados na Tabela 7.

Tabela 7: Aplicabilidade do Método Proposto - Caso 2

\begin{tabular}{|c|c|c|}
\hline \multicolumn{2}{|c|}{ Número de estados simulados } & 1080945 \\
\hline \multicolumn{2}{|c|}{ Número total de transições } & 46349 \\
\hline \multirow{6}{*}{ 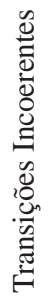 } & $R \Rightarrow M$ & $0(0.000 \%)$ \\
\hline & $R \Rightarrow H$ & $0(0.000 \%)$ \\
\hline & $M \Rightarrow H$ & $0(0.000 \%)$ \\
\hline & $H \Rightarrow M$ & $0(0.000 \%)$ \\
\hline & $H \Rightarrow R$ & $0(0.000 \%)$ \\
\hline & $M \Rightarrow R$ & $0(0.000 \%)$ \\
\hline \multicolumn{2}{|c|}{$\delta$ [oc/ano] } & 1.475 \\
\hline
\end{tabular}

Com a inclusão da representação da carga, não houve nenhuma transição incoerente entre todas as 46349 transições ocorridas durante a simulação seqüencial. Além disso, o termo $\delta$ calculado representa apenas $0.77 \%$ do índice $F(M)$, o que é bem menor que a incerteza relativa do mesmo.

Dessa forma, conclui-se que a introdução da modelagem da variação da carga não comprometeu a validade do método proposto. As hipóteses de coerência e de frequiência de transição baixa entre os estados saudável e de risco permaneceram válidas também neste caso.

\section{CONCLUSÕES}

A avaliação da confiabilidade composta sob o enfoque do bem-estar fornece informações adicionais em relação à avaliação tradicional. Além dos índices básicos referentes aos estados de risco, são também calculados novos índices que estimam a probabilidade e a freqüência com que o sistema se aproxima desses estados. Contudo, para obter essas informações adicionais, são necessárias avaliações de adequação adicionais, aumentando em muito o tempo computacional gasto. Por isso, a busca por métodos eficientes para cálculo dos índices de bem-estar tem sido tema de pesquisa recentemente.

Este trabalho contribui para essa pesquisa apresentando um método para cálculo dos índices de freqüência baseada no método da probabilidade condicional que não necessita de nenhuma análise adicional, além daquelas já gastas para classificar o estado do sistema. Esse método está baseado em duas hipóteses: o sistema de potência é coerente e a freqüência de transição entre estados de risco e saudável é pequena. Ambas as hipóteses foram verificadas para o sistema IEEEMRTS, tanto com carga constante como com representação da variação da carga anual. O método proposto calculou índices equivalentes àqueles calculados por métodos já reportados, porém com desempenho computacional superior ao da- 
queles.

Dessa forma, a avaliação da confiabilidade composta sob enfoque do bem-estar utilizando o método proposto fornece um ganho de informação em relação à avaliação tradicional, alertando sobre a margem de segurança de estados de risco do sistema, com um custo computacional reduzido em relação aos demais métodos apresentados na literatura.

\section{REFERÊNCIAS}

Allan, R., Billinton R. (2000), "Probabilistic Assessment of Power Systems", Proceedings of the IEEE, Vol. 88, No. 2, pp. 140-162.

Billinton, R. (1969), Composite System Reliability Evaluation, IEEE Transactions on Power Apparatus and Systems, Vol. PAS-88, No. 4, pp. 276-280.

Billinton, R., Allan, R. N. (1996), Reliability Evaluation of Power Systems. 2nd Ed. Plenum Press, Nova Iorque NY, EUA

Billinton, R., Billinton, B. (1997), Unit Commitment Health Analysis for Interconnecter Systems, IEEE Transactions on Power Systems, Vol. 12, No. 3, pp. 1194-1201.

Billinton, R., Billinton, B. (2005), "Incorporating WellBeing Considerations in Generating Systems Using Energy Storage", IEEE Transactions on Power Systems, Vol. 20, No. 1, pp. 225-230.

Billinton, R., Fotuhi-Firuzabad, M. (1994), "A Basic Framework for Generating System Operating Health Analysis", IEEE Transactions on Power Systems, Vol. 9, No. 3, pp. 1610-1617.

Billinton, R., Fotuhi-Firuzabad, M., Aboreshaid, S. (1997), Power System Health Analysis, Elsevier Science - Reliability Engineering and System Safety, No. 55, pp. 1-8.

Billinton, R., Karki, R. (1999), "Application of Monte Carlo Simulation to Generating System Well-being Analysis", IEEE Transactions on Power Systems, Vol. 14, No. 3, pp. 1172-1177.

Billinton, R., Khan, E. (1992), "A Security Based Approach to Composite Power System Reliability Evaluation", IEEE Transactions on Power Systems, Vol. 7, No. 1, pp. 65-72.

Billinton, R., Li, W. (1993), A System State Transition Sampling Method for Composite System Reliability Evaluation, IEEE Transactions on Power Systems, Vol. 8, No. 3, pp. 761-770.
Billinton, R., Li, W. (1994), Reliability Assessment of Electrical Power Systems Using Monte Carlo Methods. Plenum Publishing, Nova Iorque - NY, EUA

Billinton, R., Lian, G. (1994), "Composite Power System Health Analysis Using a Security Constrained Adequacy Evaluation Procedure", IEEE Transactions on Power Systems, Vol. 9, No. 2, pp. 936-941.

Borges, C. L. T., Falcão, D. M., Mello, J. C. O., Melo, A. C. G. (2001), "Composite Reliability Evaluation by Sequential Monte Carlo Simulation on Parallel and Distributed Processing Environments", IEEE Transactions on Power Systems, Vol. 16, No. 2, pp. 203-209.

CEPEL (2006), Programa NH2 - Sistema Computacional Para Análise Probabilística e Avaliação de Confiabilidade de Sistemas Elétricos, Versão 8.0, Rio de Janeiro.

Fardis, M. N., Cornell, C. A. (1981), "Analysis of Coherent Multistate Systems", IEEE Transactions on Reliability, Vol. R-30, No. 2.

Goel, L., Feng. C.. (1999), "Well-being Framework for Composite Generation and Transmission System Reliability Evaluation", IEE Proc. -Gener. Transm. Distrib., Vol. 146, No. 5, pp. 528-534.

Granville, S. (1994), "Optimal Reactive Dispatch through interior point methods", IEEE Transactions on Power Systems, Vol. 9, No. 1, pp. 136-146.

IEEE Task Force (1979), "IEEE Reliability Test System", IEEE Transactions on Power Apparatus and Systems, Vol. PAS-98, No. 6, pp. 2047-2054.

Ierusalimschy, R., Figueiredo, L. H., Celes, W. (2006), Lua 5.1 - Reference Manual, Disponível em http://www.lua.org.

Khan, E., Billinton, R. (1992), "A Hybrid Model for Quantifying Different Operating States of Composite Systems", IEEE Transactions on Power Systems, Vol. 7, No. 1, pp. 187-193.

Leite da Silva, A. M., Manso, L. A. F., Mello, J. C. O., Billinton, R. (2000), "Pseudo-Chronological Simulation for Composite Reliability Analysis with Time Varying Loads", IEEE Transactions on Power Systems, Vol. 15, No. 1, pp. 73-80.

Leite da Silva, A. M., Resende, L. C., Manso L. A. F., Billinton, R. (2004), "Well-Being Analysis for Composite Generation and Transmission Systems", IEEE Transactions on Power Systems, Vol. 19, No. 4, pp. 1763-1770. 
Manso L. A. F., Leite da Silva, A. M., (2004), "Modelagem de Cargas Variantes no Tempo na Avaliação de Confiabilidade Composta via Simulação Monte Carlo NãoSequencial", Controle \& Automação, Vol. 15, No. 1, pp. 93-100.

Manso L. A. F., Leite da Silva, A. M., Resende, L. C., Sales, W. S., Billinton R. (2004), "Avaliação da Confiabilidade Preventiva de Sistemas de Potência", IX SEPOPE, No. SP-065, pp.

Mello, J. C. O., Pereira, M. V. F., Leite da Silva, A. M. (1994), "Evaluation of Reliability Worth in Composite System Based on Pseudo-Sequential Monte Carlo Simulation”, IEEE Transactions on Power Systems, Vol. 9, No. 3, pp. 1318-1326.

Melo, A. C. G. (1990), Avaliação dos Índices de Frequência e Duração no Cálculo da Confiabilidade Composta de Sistemas de Geração e Transmissão de Grande Porte. Tese de D.Sc., Pontifícia Universidade Católica, Rio de Janeiro.

Melo, A. C. G., Pereira, M. V. F., Leite da Silva, A. M. (1992), "Frequency and Duration Calculation in Composite Generation and Transmission Reliability Evaluation", IEEE Transactions on Power Systems, Vol. 7, No. 2, pp. $469-476$.

Melo, A. C. G., Pereira, M. V. F., Leite da Silva, A. M. (1993), "A Conditional Probability Approach to the Calculation of Frequency and Duration Indices in Composite Reliability Evaluation", IEEE Transactions on Power Systems, Vol. 8, No. 3, pp. 1118-1125.

Pereira, M. V. F., Balu, N. J. (1992), Composite Generation and Transmission Reliability Evaluation, Proceedings of the IEEE, Vol. 80, No. 4, pp. 470-491.

Resende, L. C., Leite da Silva, A. M., Manso (2008), “Avaliação da Confiabilidade Convencional e Preventiva de Sistemas de Geração e Transmissão", Anais do XVII Congresso Brasileiro de Automática, Juiz de Fora MG.

Wangdee, W., Billinton, R. (2006), "Bulk Electric System Well-Being Analysis Using Sequential Monte Carlo Simulation", IEEE Transactions on Power Systems, Vol. 21, No. 1, pp. 188-193. 